\title{
eJRIEPS
}

Ejournal de la recherche sur l'intervention en éducation physique et sport

Hors-série $N^{\circ} 4$ | 2021

Actes de la $11^{\mathrm{e}}$ Biennale de l'ARIS, Liège, 25-28 février 2020

\section{Au-delà de la performance en sport scolaire : quels apports des entraîneurs à leurs sportifs ?}

Going Beyond Performance in School Sport: What is the Role of Coaches Towards their Athletes?

\section{Camille Sabourin et Martin Camiré}

\section{OpenEdition}

Journals

Édition électronique

URL : https://journals.openedition.org/ejrieps/6259

DOI : 10.4000/ejrieps.6259

ISSN : 2105-0821

Éditeur

ELLIADD

\section{Référence électronique}

Camille Sabourin et Martin Camiré, «Au-delà de la performance en sport scolaire : quels apports des entraîneurs à leurs sportifs ? », eJRIEPS [En ligne], Hors-série N 4 | 2021, mis en ligne le 10 juin 2021 consulté le 03 juillet 2021. URL : http://journals.openedition.org/ejrieps/6259 ; DOI : https://doi.org/ 10.4000/ejrieps. 6259

La revue eJRIEPS est mise à disposition selon les termes de la Creative Commons Attribution 4.0 International License. 
eJRIEPS Hors Série 4 Juillet 2021

\title{
Au-delà de la performance en sport scolaire : quels apports des entraîneurs à leurs sportifs?
}

\author{
Camille Sabourin* \& Martin Camiré* \\ * École des sciences de l'activité physique, Université d'Ottawa, Canada
}

Résumé : Le développement positif des jeunes est un mouvement de recherche basé sur les forces des jeunes dont l'objectif est de pourvoir ceux-ci d'opportunités d'apprentissage les préparant à devenir des membres productifs de la société. Dans cette optique, le sport est situé comme un contexte favorable au développement positif des jeunes, puisqu'il suscite l'acquisition d'habiletés de vie transférables à d'autres contextes. L'acquisition d'habiletés de vie est une priorité en sport scolaire; contexte permettant aux jeunes de rester actifs, se sentir connectés à leur école et développer des relations étroites avec leurs enseignants et leurs entraîneurs. Ainsi, le présent article commence par introduire les concepts de développement positif des jeunes et d'habiletés de vie, puis, dans la même lignée, dresse un portrait du sport scolaire au Canada. Ensuite, le rôle de l'entraîneur est abordé, ainsi que l'apport de la formation d'entraîneur centrée sur l'enseignement des habiletés de vie. L'article conclu avec une vision de l'avenir concernant le rôle de l'entraîneur scolaire en tant qu'acteur clé pour promouvoir la justice sociale soutenant l'égalité des droits et une solidarité collective.

Mots clés : développement positif des jeunes; habiletés de vie; formation; justice sociale.

Going Beyond Performance in School Sport: What is the Role of Coaches Towards their Athletes?

Summary: Positive youth development is an approach to research based on the strengths of youth. The aim is to provide youth with learning opportunities that prepare them to become productive members of society. From this perspective, sport is seen as a favorable context for positive youth development, given that it facilitates the acquisition of life skills transferable to other contexts. The acquisition of life skills is a priority in school sport, a context that allows young people to stay active, feel connected to their school, and develop close relationships with their teachers and coaches. Thus, the present article starts by introducing the concepts of positive youth development and life skills, then, in the same vein, presents 
a portrait of school sport in Canada. The role of the coach is discussed, as well as the contribution of life skills-specific coach education. The article concludes with a look toward the future in terms of the role the school coach can play in promoting social justice by supporting equal rights and promoting a collective solidarity.

Key words: positive youth development; life skills; training; social justice.

\section{Le développement positif des jeunes en sport}

Fondé sur les principes de la théorie des systèmes de développement relationnel (Geldhof et al., 2013 ; Snyder \& Lopez, 2002), le développement positif des jeunes (DPJ) est une approche situant les jeunes comme des «ressources à développer » plutôt que des « problèmes à gérer » (Lerner, 2002). Selon cette approche, un développement optimal se définit par l'acquisition des habiletés cognitives, sociales, affectives et intellectuelles nécessaires pour devenir de bons citoyens (Weiss \& Wiese-Bjornstal, 2009). Trois conditions clés, lorsqu'elles sont combinées, accentueraient le DPJ, soient (1) la présence de relations positives et durables avec des adultes, (2) la participation à des activités facilitant l'acquisition d'habiletés de vie (HV) et (3) la participation à des activités communautaires valorisantes. Somme toute, depuis le début des années 2000, le DPJ est l'une des approches les plus utilisées pour conceptualiser le développement psychosocial des jeunes en recherche en sport (Holt, 2016).

Le sport organisé a été positionné comme un contexte favorable au DPJ en raison de sa structure, sa compétitivité et son volet social (p. ex., Gould \& Carson, 2008; Larson, 2000). De façon générale, les jeunes sont motivés à pratiquer des sports et des études ont démontré que la participation sportive peut avoir des effets positifs sur l'estime de soi, la confiance, le sentiment d'appartenance et la réussite scolaire (Camiré, Trudel \& Forneris, 2009a ; Fraser-Thomas et al., 2005 ; Holt \& Neely, 2011). En revanche, malgré ces apports positifs, la participation sportive peut potentiellement générer des expériences négatives, telles que la promotion de normes sociales indésirables (Eccles \& Barber, 1999 ; Mahoney, 2000) engendrant stress et anxiété (Scanlan et al., 2005 ; Smoll \& Smith, 1996). Conséquemment, la façon dont le contexte sportif est structuré a une influence directe et considérable sur l'expérience des jeunes, ainsi que sur les différentes HV qui sont développées (Camiré, Trudel \& Forneris, 2009b; Holt, 2016). 


\section{Les habiletés de vie}

Parmi les nombreuses approches de recherche en sport, telles que les techniques sportives en relation à leur didactique (Lafont, 2002), l'étude des compétences de l'expert (Macquet \& Fleurance, 2006), la santé mentale et le bien-être en sport (Leavey \& Breslin, 2019), l'étude des HV attire de plus en plus l'attention des chercheurs. Les HV en sport sont définies comme étant des habiletés psychosociales telles que la fixation d'objectifs, la gestion des émotions et la persévérance pouvant être développées en sport et ensuite transférées dans des contextes non sportifs (Gould \& Carson, 2008). Le transfert des HV offrirait aux jeunes une gamme d'outils leur permettant de faire face aux exigences et aux défis rencontrés à l'école, à la maison, au travail et dans la communauté (Hodge \& Danish, 1999). La notion du transfert signifie qu'une habileté acquise en sport se doit d'être transférée au-delà du sport pour être considérée comme une HV. La notion du transfert des HV fut définie par Pierce et al. (2017) comme étant :

Un processus continu par lequel une personne développe ou apprend et intériorise une habileté (c.-à-d., compétences psychosociales, connaissances, disposition, construction d'identité ou transformation) en sport, puis fait l'expérience d'un changement interne par l'application de cette habileté dans un ou plusieurs domaines de vie au-delà du contexte où elle a été initialement apprise. (p. 194)

Cette définition comprend cinq éléments clés qui permettent de bien cerner le concept des HV. Dans le présent article, le terme « apprenant » réfère à tous les sportifs en processus d'apprentissage/acquisition d'HV et est utilisé afin de faciliter la lecture.

Premièrement, l'apprentissage est un processus continu par lequel l'apprenant apprend une HV dans un contexte et l'utilise ensuite dans un ou plusieurs autres contextes. Par conséquent, le transfert représente le processus intermédiaire entre l'apprentissage dans un contexte et l'utilisation dans un autre. Définir le transfert comme un processus implique qu'il se produit dans le temps selon les opportunités de transfert qui se présentent à l'apprenant (Danish et al., 1993 ; Lee \& Martinek, 2013). Le transfert peut donc se manifester immédiatement ou tardivement.

Deuxièmement, le potentiel d'apprentissage et de transfert réside dans l'apprenant (Bereiter, 1995). Comme le suggèrent des études récentes (Chinkov \& Holt, 2016 ; Pierce et al., 2016), ces processus se produisent lorsqu'il y a présence d'environnements propices à l'apprentissage et au transfert. L'apprenant doit tout d'abord internaliser une HV (c.-à-d., l'intégrer consciemment ou inconsciemment dans son concept de soi) pour ensuite la 
généraliser dans d'autres contextes (Hodge et al., 2013). Le transfert est donc un processus situé au croisement de l'internalisation et de la généralisation. Puisque les apprenants entrent dans le contexte sportif avec un certain bagage d'expériences et de connaissances (Gould \& Carson, 2008), l'apprentissage en sport peut s'opérer de par (1) l'acquisition de nouvelles HV ou (2) le raffinement d'HV existantes (Kendellen \& Camiré, 2015a). Le transfert peut ensuite se manifester différemment pour chaque apprenant en fonction de son répertoire d'expériences et de connaissances en constante évolution.

Troisièmement, les HV englobent les compétences psychosociales, les connaissances, les dispositions et les constructions ou transformations identitaires. Les compétences psychosociales peuvent être interpersonnelles (p. ex., communiquer efficacement) ou intrapersonnelles (p. ex., gérer son temps ; Gould \& Westfall, 2013 ; Petitpas et al., 2005). Les connaissances sont définies comme conceptuelles (c.-à-d., savoir cela), procédurales (c.-à-d., savoir comment), stratégiques (c.-à-d., savoir pourquoi) et tacites (c.-à-d., connaissances expérientielles personnelles; Leberman et al., 2006). Les dispositions (p. ex., la compétitivité, le perfectionnisme) sont définies comme des schèmes acquis de perceptions, de pensées et d'actions (Bourdieu, 1990). Bien que les dispositions puissent être considérées innées, des travaux empiriques (p. ex., Jones \& Lavallee, 2009) ont démontré que pendant l'enfance et l'adolescence, elles peuvent être modelées dans le contexte sportif en réponse aux conditions environnementales. Les constructions ou transformations identitaires peuvent émaner de la participation sportive selon le degré auquel l'apprenant s'identifie au rôle d'athlète dans le cadre de son concept de soi global (Brewer et al., 1993). Cette représentation des HV est cohérente avec l'approche transformationnelle de Hager et Hodkinson (2009), d'où l'apprentissage et le transfert sont constitués comme des constructions/reconstructions internes continuelles.

Quatrièmement, l'apprentissage des HV peut se produire dans de nombreux contextes, y compris le sport (Lee \& Martinek, 2013). Les apprenants passent quotidiennement d'un contexte à l'autre et par conséquent, ceux qui s'intéressent à l'étude des HV doivent considérer le sport comme étant un des nombreux contextes d'apprentissage (p. ex., l'école, la famille) permettant aux apprenants d'apprendre des HV.

Cinquièmement, le transfert des HV peut se produire dans une variété de contextes. Chaque contexte a son propre ensemble de conditions facilitant ou limitant le transfert des HV. Cependant, étant donné que le processus de transfert réside dans l'apprenant, ces conditions de transfert se construisent cognitivement au sein de celui-ci et sont davantage des barrières perceptives que des entités objectivement distinctes (Hager \& Hodkinson, 2009). Donc, les conditions facilitant ou limitant le transfert dans différents contextes ne sont 
pas des marqueurs rigides, mais plutôt des conditions interprétées de différentes façons par différents apprenants. En somme, l'émergence du DPJ en sport au cours des dernières années a permis de passer d'une approche déficitaire à une mise en lumière de l'importance d'enseigner des HV en sport. Selon plusieurs chercheurs (Beauchamps et al., 2018 ; Danish et al., 2005), le contexte du sport scolaire est particulièrement propice à la pratique sportive puisque les jeunes passent une grande partie de leur journée à l'école.

\section{Le sport scolaire}

Le sport scolaire est un contexte pouvant favoriser l'apprentissage des $\mathrm{HV}$ et ainsi permettre aux jeunes d'acquérir les outils nécessaires pour devenir des citoyens engagés dans la société (Camiré et al., 2009a). Le sport scolaire fait référence au sport organisé par les écoles et est pratiqué en dehors des heures de classe. Les jeunes font partie d'équipes sportives qui concourent dans des ligues interscolaires menant à des championnats de fin de saison (Camiré \& Kendellen, 2016). L'aspect organisé, compétitif et interscolaire du sport scolaire le différencie d'autres activités physiques que l'on retrouve à l'école, comme les sports intra-muros, les cours d'éducation physique ou le jeu libre (Camiré, 2014). Au Canada, le sport scolaire offre aux jeunes âgés approximativement de 13 à 18 ans la possibilité de s'engager dans une variété de sports d'équipe et individuels. En règle générale, une saison sportive dure quelques mois (saison d'automne, d'hiver et de printemps), permettant ainsi aux jeunes de pratiquer plusieurs sports durant l'année scolaire. Ainsi, pour des centaines de milliers de jeunes, le sport scolaire fait partie intégrante de leur expérience éducative, un milieu dans lequel ils peuvent développer un sentiment d'appartenance à leur équipe et à leur école tout en forgeant des liens avec des coéquipiers, des entraîneurs et d'autres personnes impliquées (Camiré, 2014).

Selon la fédération nationale canadienne Sport scolaire Canada, l'objectif principal de la participation sportive dans les écoles est de promouvoir l'esprit sportif, la citoyenneté et le développement global des élèves-athlètes par le sport. Le système sportif scolaire canadien compte plus de 52000 bénévoles qui entraînent 750000 jeunes pratiquant des sports dans 3200 écoles à travers le pays (Sport scolaire Canada, 2020). Cependant, un pourcentage infime d'élèves-athlètes se verront poursuivre leur sport au niveau universitaire. En effet, selon les statistiques de la National Collegiate Athletic Association (NCAA, 2018), organisme réglementant le sport universitaire américain, $28 \%$ des athlètes internationaux inscrits auprès de la NCAA sont d'origines canadiennes. Leurs statistiques démontrent que moins de $5 \%$ des jeunes ayant pris part au sport scolaire lorsqu'ils étaient adolescents chemineront vers un programme de division 1 universitaire de la NCAA. Peu d'entre eux 
(variant selon différents facteurs, incluant le type de sport) se verront percer au sein des ligues professionnelles. Par conséquent, le sport scolaire se positionne en tant que contexte favorisant à la fois le développement sportif et global de tous les jeunes et non un contexte priorisant uniquement la performance de l'élite vers les rangs professionnels.

Afin de distinguer différents types de programmes sportifs, Coalter (2007) a mis au point une classification (c.-à-d., Sport, Sport Plus et Plus Sport) en fonction de leurs objectifs développementaux. Cette classification permet de comprendre où se situe le sport scolaire en matière de développement comparativement à d'autres contextes sportifs. Les programmes Sport sont des programmes traditionnels à travers lesquels les jeunes apprennent des habiletés physiques, techniques et tactiques. De façon générale, ces programmes priorisent la performance et le cheminement des jeunes vers de plus hauts niveaux de compétition. Ceux-ci se distinguent des programmes Sport Plus, où le sport est une caractéristique essentielle, mais adaptée pour maximiser le développement psychosocial, les habiletés motrices, ainsi que les HV. Finalement, les programmes Plus Sport constatent que le sport n'est pas une fin en soi, mais sert plutôt d'outil pour mettre de l'avant le développement psychologique, social et culturel des participants. Le sport agit donc à titre de vecteur de croissance humaine, la priorité étant accordée aux résultats non sportifs (p. ex., éducation, sensibilisation, changement de comportement; Coalter, 2007). $\mathrm{Au}$ sein des programmes Plus Sport, la compétition est délibérément atténuée afin d'optimiser la réalisation d'objectifs liés au développement des jeunes. Certaines études, notamment réalisées au Québec en langue française, ont évalué des programmes Plus Sport vu leur portée sur le DPJ et le transfert d'HV (p. ex., Simard, 2014 ; Trottier \& Robitaille, 2014). Ainsi, et de par la classification de Coalter (2007), le sport scolaire se situe comme un programme répondant aux objectifs de Sport Plus.

\section{Le développement des jeunes par le sport scolaire}

Les résultats de recherches ont démontré que les jeunes pratiquant des sports scolaires ont généralement de meilleurs résultats scolaires, des aspirations professionnelles plus élevées, et une image de soi plus positive que les non-participants (Clark et al., 2015 ; Fox et al., 2010 ; Harrison \& Narayan, 2003 ; Marsh \& Kleitman, 2003). Sur le plan de la santé mentale, la participation aux sports scolaires est liée à un meilleur bien-être et à une plus grande résilience chez les jeunes (Appelqvist-Schmidlechner et al., 2017 ; Ho et al., 2017). Des études ont également démontré comment les jeunes pratiquant des sports scolaires ont généralement des taux plus faibles de détresse émotionnelle, d'abandon scolaire, de 
comportements suicidaires et de problèmes alimentaires comparativement aux nonparticipants (Harrison \& Narayan, 2003 ; Neely \& Vaquera, 2017 ; Steiner et al., 2000).

Cependant, il importe de préciser que le sport scolaire n'est pas une panacée (Coakley, 2011) et que la participation des jeunes est liée à des taux plus élevés de consommation d'alcool (Eccles et al., 2003 ; Green et al., 2014 ; Kwan et al., 2014), à une plus grande probabilité de blessures (Crane \& Temple, 2015) et même au «burnout » (DiFiori et al., 2014). Des études qualitatives ont démontré comment des jeunes ont été confrontés à des comportements inappropriés provenant de leurs pairs et de leurs entraîneurs (Dworkin \& Larson, 2006) en plus de ressentir une pression excessive de leurs parents (Fraser-Thomas \& Côté, 2009).

Certains chercheurs se sont intéressés à comprendre pourquoi les entraîneurs sont dans des positions privilégiées pour maximiser les aspects positifs et réduire les aspects négatifs de la participation dans les sports scolaires. Premièrement, en raison de la configuration compétitive et/ou coopérative du sport, les entraîneurs ont plusieurs opportunités pour favoriser l'acquisition/le raffinement d'HV (p. ex., l'éthique de travail, le travail d'équipe, la persévérance) aidant les jeunes à confronter les événements stressants de leur vie (Bean \& Forneris, 2016 ; Broh, 2002). Deuxièmement, la participation aux sports scolaires étant fondamentalement sociale, les entraîneurs procurent aux jeunes des opportunités pour développer leurs réseaux et amasser du capital social (Van Boekel et al., 2016). Troisièmement, en raison des nombreuses heures passées avec les jeunes avant, pendant et après les compétitions, les entraîneurs sont en mesure de forger des relations significatives avec eux. Ainsi, les entraîneurs sont en position d'influence «complémentaire»sur les jeunes ayant de bonnes situations familiales/sociales ou d'influence « compensatoire » sur les jeunes en situations familiales/sociales défavorables (Bowers et al., 2014; Camiré, 2015a). La prochaine section examine, d'une perspective développementale, le rôle de l'entraîneur.

\section{Le rôle de l'entraîneur}

\subsection{Le climat}

L'entraîneur est un acteur clé du contexte sportif puisqu'il est responsable pour procurer des expériences sportives de qualité. Des études antérieures ont indiqué que le développement des jeunes (autant positif que négatif) résultant de la participation dans les sports scolaires est en grande partie influencé par le climat généré par l'entraîneur (Buford May, 2001 ; Dworkin \& Larson, 2006 ; Jewett et al., 2014 ; Van Boekel et al., 2016). Lorsque l'entraîneur offre des instructions appropriées, établit des relations de qualité, satisfait les besoins 
psychologiques fondamentaux des jeunes et enseigne des HV, il est en position idéale pour aider les jeunes à exceller dans toutes les sphères de leur vie (Bailey et al., 2009 ; Carson Sackett \& Gano-Overway, 2017).

Un élément à considérer à l'échelon du sport scolaire canadien est que les postes d'entraîneurs sont assumés en grande partie par des enseignants qui entraînent des équipes sportives à leur école bénévolement. Ainsi, ces enseignants ne sont pas professionnellement obligés à entraîner ; ils entraînent parce qu'ils ont une passion pour le sport et ressentent une obligation morale de bonifier la vie étudiante des jeunes par le sport. Camiré (2015a, 2015b) a examiné les conditions de travail des individus assumant le double rôle d'enseignant-entraîneur scolaire. Les résultats ont indiqué qu'occuper à la fois le rôle d'enseignant et d'entraîneur permet d'accroître le nombre d'interactions avec les élèves, engendrant des relations mutuelles significatives basées sur la confiance et le respect, approvisionnant ainsi le DPJ (Camiré, 2015a). Cependant, les responsabilités associées au double rôle d'enseignant-entraîneur mènent souvent à une surcharge de travail, agissant sur la capacité à gérer les obligations en sport, à l'école et à la maison (Camiré, 2015b). Somme toute, le double rôle d'enseignant-entraîneur offre des conditions de travail pouvant optimiser le DPJ, mais souvent au prix de compromettre l'équilibre travail-famille.

\subsection{L'influence}

Des études ont examiné les bénéfices perçus par les entraîneurs quant à la participation des jeunes en sport scolaire. Les résultats indiquent qu'ils considèrent le développement physique, psychologique et social des jeunes comme l'objectif principal du sport scolaire (Gould et al., 2006). De plus, les entraîneurs auraient des attentes élevées à l'égard du sport scolaire dans le but de faciliter le DPJ et de promouvoir l'acquisition d'HV.

Par contre, et malgré ces attentes élevées, bien des entraîneurs n'enseignent pas les HV de façon intentionnelle. En effet, des études (p. ex., Holt et al., 2008 ; Lacroix et al., 2008) ont démontré que la plupart des entraîneurs auraient peu de stratégies concrètes pour enseigner les HV, certains croyant même que ces stratégies concrètes ne seraient pas nécessaires pour promouvoir le DPJ (Bean \& Forneris, 2017). II demeure que des études ont mis en lumière que certains entraîneurs, surtout ceux possédant plusieurs années d'expérience, enseigneraient délibérément des HV aux jeunes. Deux études menées auprès de dix entraîneurs de football scolaire hautement expérimentés (Collins et al., 2009 ; Gould et al., 2007) ont permis de constater que ces entraîneurs concevaient le DPJ et la performance d'une approche inclusive. Ces entraîneurs utilisaient des stratégies tangibles pour enseigner des HV comme offrir des rétroactions personnalisées, promouvoir des 
d'objectifs réalistes et conscientiser les jeunes sur le lien crucial entre le football et la vie. Pour leur part, Camiré et al. (2012) ont étudié la manière dont les entraîneurs scolaires expérimentés ont appris à faciliter le DPJ et à favoriser l'apprentissage et le transfert d'HV. Les résultats ont révélé que ces entraîneurs étaient des individus très motivés profitant de situations d'apprentissage à leur disposition pour bonifier leur enseignement d'HV. De plus, ces entraîneurs offraient fréquemment à leurs athlètes des opportunités pour pratiquer leurs HV lors de matchs et d'entraînements.

Somme toute, bien que les entraîneurs sont des acteurs influents dans le contexte du sport scolaire, plus de preuves empiriques sont nécessaires afin de démontrer de façon exhaustive comment ils favorisent le DPJ. Des études futures devraient examiner de plus près comment l'acte d'entraîner est un mécanisme essentiel lié au DPJ dans le sport scolaire. Plus précisément, ces études pourraient évaluer comment différentes approches (p. ex., culturaliste, transversale et méthodologique) peuvent jumeler, de façon inclusive ou non, la formation aux HV avec les acquisitions techniques/tactiques, tout en considérant les préoccupations de performance présentent dans les structures sportives de plusieurs pays. Finalement, un autre mécanisme à considérer comme voie de recherche est la formation des entraîneurs en matière de DPJ et d'HV.

\section{La formation des entraîneurs}

Une récente étude de Newman et al. (2016) a démontré que les entraîneurs considèrent les HV comme des habiletés importantes à acquérir par le biais du sport et estiment que former les entraîneurs à enseigner les HV devrait être une priorité. Par contre, selon Vella et al. (2013), la plupart des formations pour les entraîneurs négligent le DPJ. Ainsi, pour combler ces lacunes, des chercheurs ont développé des formations spécialisées pour former les entraîneurs à promouvoir le DPJ et les HV (Harwood et al., 2015 ; Strachan et al., 2016 ; Vella et al., 2013), incluant certaines formations spécifiques au sport scolaire (p. ex., Camiré et al., 2018 ; Ferris et al., 2016). En règle générale, les évaluations de ces formations spécialisées ont offert des résultats positifs, les entraîneurs indiquant une meilleure compréhension du DPJ et des HV. De plus, les entraîneurs ont indiqué comment les formations ont augmenté l'ensemble de leurs connaissances, amélioré leur autoréflexion, et augmenté leur confiance à enseigner des HV. Une formation en particulier, Entraîner les habiletés de vie, est détaillée, relatant de sa conception puis de son évaluation.

\subsection{Entraîner les habiletés de vie}




\subsubsection{La conception}

La formation Entraîner les habiletés de vie fut élaborée en collaboration avec Sport scolaire Canada dans le cadre d'un projet de recherche financé par le Conseil de recherches en sciences humaines du Canada. Lors de la conception initiale, le DPJ a servi de fondation théorique afin de promouvoir une approche axée sur les HV. Conformément aux trois conditions clés du DPJ, la formation Entraîner les habiletés de vie a été conçue pour améliorer la capacité des entraîneurs à (1) optimiser la relation entraîneur-athlète, (2) favoriser le développement des HV en sport et (3) faciliter le transfert de ces HV dans des contextes hors sport. La formation a été créée sous forme d'un atelier de courte durée, accompagné d'un manuel de l'entraîneur, vu les contraintes de temps auxquelles les entraîneurs scolaires se voient confrontés (Camiré et al., 2016). Des études antérieures ont démontré que les formations de courte durée peuvent mener à des résultats d'apprentissage favorables ( $p$. ex., entraîneurs plus solidaires, amusants, axés sur la matière et moins punitifs) comme l'indique les formations Coach Effectiveness Training et Mastery Approach to Coaching (Smith et al., 1995 ; Smith et al., 2007).

Le but ultime de la formation est de sensibiliser les entraîneurs au potentiel d'utiliser le sport scolaire comme contexte propice à l'enseignement des HV. Le manuel de l'entraîneur a été conçu pour favoriser la réflexion et offrir des stratégies concrètes permettant aux entraîneurs d'enseigner délibérément des HV. Sur le plan pédagogique, une approche centrée sur l'apprenant a été adoptée lors des ateliers, en utilisant des aspects de l'apprentissage par problèmes (Savery, 2015). Les ateliers sont divisés en cinq modules.

Dans le premier module, à titre d'introduction, le rôle privilégié de l'entraîneur scolaire est illustré (Petitpas et al., 2005), suivi des principaux constats du DPJ (Lerner et al., 2005) et d'une définition des HV (Danish et al., 2004).

Dans le deuxième module, l'importance de la philosophie d'entraînement est abordée sur la base d'études menées dans le contexte du sport scolaire (Collins et al., 2009). À l'aide du manuel de l'entraîneur, une activité «think-pair-share » est suggérée, durant laquelle les participants travaillent individuellement pendant 10 minutes sur leur philosophie en répondant à trois questions: (1) Pour quelles raisons j'entraîne ?; (2) Qu'est-ce que j'aimerais que mes athlètes apprennent de leur participation au sport scolaire ? et (3) Mes athlètes sont-ils de meilleures personnes de par nos interactions? Par la suite, les participants se regroupent en pairs pour discuter de leurs trois réponses et des défis auxquels ils font face dans la mise en œuvre de leur philosophie. Les principes philosophiques sont ensuite partagés devant le groupe entier, les participants étant fortement encouragés à affiner leur philosophie d'entraînement à la suite de l'atelier. 
Dans le troisième module, l'accent est placé sur la relation entraîneur-athlète selon le modèle de Jowett (2007) qui suggère aux entraîneurs de cultiver la proximité, l'engagement, la complémentarité et la co-orientation avec leurs athlètes. Les participants visionnent et discutent une vidéo d'un entraîneur scolaire entretenant des relations étroites avec ses athlètes. Les participants reçoivent ensuite des stratégies concrètes pour promouvoir les besoins psychologiques fondamentaux de leurs athlètes, soit l'autonomie, l'affiliation et la compétence (Ryan \& Deci, 2000) en plus d'exemples de rétroactions favorisant l'autonomie de leurs athlètes (Carpentier \& Mageau, 2016). Les besoins psychologiques fondamentaux sont discutés en détail avec les participants, mettant en lumière leur rôle sur le DPJ (Camiré et al., 2019).

Dans le quatrième module, les participants reçoivent plusieurs exemples d'activités facilitant le développement des HV chez les jeunes en sport scolaire, basé sur l'approche SAFE de Durlak et al. (2010). Selon cette approche, les activités enseignées aux jeunes devraient être séquencées, actives, ciblées et explicites afin de promouvoir de façon optimale l'apprentissage des HV en sport scolaire.

Dans le cinquième module, les participants reçoivent une définition du transfert des HV et sont exposés aux facteurs contextuels et psychologiques influençant le transfert selon le modèle de Pierce et al. (2017). Ensuite, plusieurs stratégies (Pierce et al., 2018) facilitant le transfert des HV dans différents contextes de vie sont présentées. L'atelier se termine en encourageant les participants à adapter les stratégies de la formation Entraîner les habiletés de vie à leur propre réalité d'entraîneur.

\subsubsection{L'évaluation}

La formation Entraîner les habiletés de vie a été évalué en trois temps. Premièrement, une étude pilote utilisant un devis qualitatif a permis d'explorer la perspective des participants concernant l'utilité de la formation (Camiré et al., 2018). Les ateliers de l'étude pilote ont été livrés en personne à 68 entraîneurs scolaires canadiens qui ont pris part à l'un des six ateliers de trois heures (c.-à-d., trois ateliers en anglais, trois ateliers en français). Sur ces 68 entraîneurs, 10 ont participé à des entretiens individuels semi-structurés. Selon les résultats, les participants ont exprimé avoir appris l'importance (1) de la relation entraîneurathlète, (2) d'enseigner des HV en sport scolaire et (3) d'utiliser des stratégies ciblant délibérément le développement et le transfert des HV.

Deuxièmement, à la suite de l'étude pilote, la formation Entraîner les habiletés de vie a été transformé en une formation en ligne (offerte sur www.schoolcoach.ca) comprenant également cinq modules. Un essai contrôlé randomisé a permis d'évaluer l'efficacité de la 
formation pour aider les entraîneurs à créer des environnements propices à l'enseignement des HV (Camiré et al., 2020). Au total, 1238 entraîneurs canadiens ont complété les mesures pré-intervention portant sur la relation entraîneur-athlète, les comportements interpersonnels des entraîneurs et l'enseignement des HV. Les participants ont ensuite été randomisés soit à un groupe expérimental, un groupe liste d'attente ou un groupe contrôle. Dû à la complexité des essais contrôlés randomisés et aux limites associées aux études réalisées en ligne, un échantillon final de 285 participants a terminé l'essai, soit 36 dans le groupe expérimental, 58 dans le groupe liste d'attente et 191 dans le groupe contrôle. En utilisant des analyses factorielles de mesures répétées $3 \times 3$, les résultats ont démontré un effet principal intra-sujet non significatif pour le temps, le groupe ou l'interaction entre le temps et le groupe pour les trois variables dépendantes. Bien que les résultats ne soient pas statistiquement significatifs, une analyse visuelle a indiqué des changements directionnels positifs pour les trois variables dépendantes, avec des augmentations des scores moyens observés pour le groupe expérimental et le groupe liste d'attente après avoir terminé le programme (Camiré et al., 2020).

Troisièmement, dans le but d'en connaître davantage sur les influences à plus long terme de la formation Entraîner les habiletés de vie, un suivi d'un an a été mené utilisant un devis comparatif causal prospectif 2 × 2 (Turgeon et al., 2020). Les 285 entraîneurs ayant complété l'essai contrôlé randomisé de Camiré et al. (2020) ont été invités à compléter les mesures sur la relation entraîneur-athlète, les comportements interpersonnels des entraîneurs et l'enseignement des HV un an après l'essai. L'échantillon final fut composé de 64 participants. Les résultats comparant les scores pré-intervention et les scores du suivi d'un an ont permis de constater que la relation entraîneur-athlète et les comportements interpersonnels des entraîneurs ont augmenté de façon significative pour le groupe intervention et ont diminué de façon significative pour le groupe témoin. Aucune différence significative n'a été rapportée entre les scores pré-intervention et les scores du suivi d'un an sur l'enseignement des HV. En somme, les résultats du suivi suggèrent que la formation Entraîner les habiletés de vie peut aider les entraîneurs à optimiser leur utilisation de comportements relationnels sains avec leurs athlètes. Cependant, il est nécessaire de mentionner que ces résultats ne permettent de savoir avec certitude s'il y eu présence d'un transfert d'apprentissage au moment de l'étude. Ainsi, en guise d'avenues futures de recherche, il serait pertinent d'évaluer, à l'aide d'observations, l'efficacité et le rendement des entraîneurs formés à enseigner des HV. 


\section{Un regard critique vers l'avenir : Quels apports des entraîneurs du sport scolaire en matière de justice sociale?}

Vu les mouvements récents (p. ex., la vie des Noirs compte, \#MeToo, grève étudiante pour le climat) ayant mobilisé de nombreux citoyens, nous nous devons d'examiner comment le sport scolaire, en tant que pratique sociale populaire dans certains pays, peut encourager un dialogue sain quant aux questions de justice sociale. La justice sociale est un concept polyvalent (Walton-Fisette \& Sutherland, 2018) mieux compris sous ses multiples facettes : (1) philosophique (c.-à-d., le sens de la justice), (2) pratique (c.-à-d., à quoi ressemble la justice sociale sur le terrain), (3) ethnographique (c.-à-d., les expériences vécues de ceux qui vivent la justice/l'injustice), (4) théorique (c.-à-d., les positions sur les différents mouvements sociaux) et (5) démocratique (c.-à-d., la répartition de l'oppression). La justice sociale implique donc une lutte vers l'égalité pour tous en permettant l'accès aux ressources, aux services et à l'information à tous les citoyens (Newman et al., 2019).

Le DPJ et les HV, à titre de cadres conceptuels, demeurent répandus dans le monde de la recherche en sport chez les jeunes. Cependant, certains chercheurs ont soulevé des inquiétudes, signalant les dangers de faire des louanges des vertus du sport (Coalter, 2010) en plus de réduire ce que les jeunes apprennent en sport à des HV souvent liées à la production économique (Ronkainen et al., 2020). Coakley (2016) a situé le DPJ dans une idéologie néolibérale promouvant des valeurs capitalistes où les jeunes sont socialisés à être productifs et employables. Kochanek et Erickson (2020) ont critiqué le cadre du DPJ et ont plutôt envisagé comment nous pourrions réinventer le rôle de l'entraîneur sportif d'un angle plus critique en matière de justice sociale. Les auteurs ont postulé comment les entraîneurs pourraient contribuer à remettre en question les valeurs capitalistes associées à une vision restreinte du développement des jeunes en sport.

Cependant, il importe de constater que les entraîneurs scolaires sont en grande partie des hommes blancs hétérosexuels de classe moyenne (Newman et al., 2020). Leurs pratiques hétéronormatives marginaliseraient souvent certaines populations de jeunes, les rendant moins susceptibles de participer au sport scolaire ou les rendant inconfortables lorsqu'ils y participent (Kulick et al., 2019). Appuyant la remise en question prônée par Kochanek et Erickson (2020), Newman et al. (2020) ont interrogé 122 entraîneurs majoritairement masculins $(80 \%)$ et blancs $(88 \%)$ et ont constaté que peu d'entre eux considéraient la santé mentale $(6,5 \%)$, la diversité (1,9\%), les handicaps physiques $(1,0 \%)$ et les enjeux des personnes LGTB $(0,0 \%)$ comme des notions importantes à aborder en sport, démontrant ainsi que les inégalités demeurent largement invisibles aux yeux des personnes en position de privilège (Mac Intosh et al., 2020). 
Bien que le sport offre des opportunités distinctes pour promouvoir la justice sociale (Cunningham et al., 2019), il importe de reconnaître qu'il est également un site privilégié de résistance en raison des multiples pratiques discursives qui permettent de maintenir le statu quo (Spaaij et al., 2020). Dans le but de précipiter le changement social, les entraîneurs devraient aider les jeunes à se créer pour eux-mêmes une participation sociale qui suscite le changement. Les entraîneurs se doivent de mettre en confiance et d'outiller les jeunes à agir à titre d'agents de justice sociale puisque le soutien des entraîneurs a été démontré comme étant crucial pour supporter et façonner l'activisme des jeunes (Fuller \& Agyemang, 2018). Selon Mac Intosh et al. (2020), des études futures devraient examiner de plus près la perspective des entraîneurs en matière de justice sociale et explorer comment ils se voient aider les jeunes à devenir activistes. Une prochaine étape cruciale en recherche consiste donc à examiner comment les entraîneurs peuvent créer des milieux permettant aux jeunes d'utiliser leur voix pour promouvoir la justice sociale. De tels efforts exigent que l'on réinvente le rôle de l'entraîneur scolaire dans une optique de justice sociale.

Sur la base des arguments avancés précédemment, il importe d'examiner comment les entraîneurs peuvent promouvoir la justice sociale dans le sport scolaire. Les critiques ( $p$. ex., Kochanek \& Erickson, 2020 ; Ronkainen et al., 2020) s'accumulent et démontrent comment le DPJ et les HV ont des limites conceptuelles qui réduisent le large éventail d'apprentissages et de contributions sociales pouvant découler de la participation au sport scolaire. Ces limites restreignent la façon dont nous imaginons le rôle de l'entraîneur scolaire et l'influence qu'il peut exercer en abordant des enjeux de justice sociale.

Réinventer le rôle de l'entraîneur scolaire est nécessaire dans nos sociétés pour confronter le racisme, le colonialisme, le sexisme et les autres problèmes sociaux qui depuis trop longtemps sont enracinés dans le monde du sport (Hennig et al., 2020). Des études mettant en lumière la justice sociale sont donc essentielles pour renforcir nos communautés (Jones et al., 2020) et soutenir une réelle collaboration intersectorielle entre les parties prenantes (D’Angelo et al., 2020) du système de sport scolaire (p. ex., les entraîneurs, les administrateurs et les parents). Les résultats de ces études permettront de déterminer si les procédures, les règlements, les politiques et les structures de gouvernance du système de sport scolaire devraient/pourraient être modifiés pour aborder les grandes questions de justice sociale du présent et de l'avenir. Concrètement, ces initiatives pourraient aider à remédier de manière significative à la pénurie de personnes noires, de personnes de couleur non noires et de personnes autochtones occupant des postes de leadership clés dans le système de sport scolaire (Heroux \& Stashin, 2020). 


\section{Bibliographie}

Appelqvist-Schmidlechner, K., Vaara, J., Hakkinen, A., Vasankari, T., Makinen, J., Mantysaari, M., \& Kyrolainen, H. (2017). Relationships between youth sports participation and mental health in young adulthood among Finnish males. American Journal of Health Promotion, 32(7), 1502-1509. https://doi.org/10.1177/0890117117746336

Bailey, R., Armour, K., Kirk, D., Jess, M., Pickup, I., \& Sandford, R. (2009). The educational benefits claimed for physical education and school sport: An academic review. Research Papers in Education, 24(1), 1-27. https://doi.org/10.1080/ 02671520701809817

Bean, C. \& Forneris, T. (2016). Examining the importance of intentionally structuring the youth sport context to facilitate positive youth development. Journal of Applied Sport Psychology, 28(4), 410-425. https://doi.org/10.1080/10413200.2016.1164764

Bean, C., \& Forneris, T. (2017). Is life skill development a by-product of sport participation? Perceptions of youth sport coaches. Journal of Applied Sport Psychology, 29(2), 234-250. https://doi.org/10.1080/10413200.2016.1231723

Beauchamp, M. R., Puterman, E., \& Lubans, D. R. (2018). Physical activity and mental health in late adolescence. JAMA Psychiatry, 75(6), 543-544. https://doi.org/10.1001/jamapsychiatry.2018.0385

Bereiter, C. (1995). A dispositional view of transfer. In A. McKeough, J. Lupart, \& A. Marini. (Eds.), Teaching for transfer. Fostering generalization in learning (p. 21-34). Lawrence Erlbaum.

Bourdieu, P. (1990). The logic of practice. Stanford University Press.

Bowers, E. P., Johnson, S.K., Buckingham, M.H., Gasca, S., Warren, D.J., Lerner, J.V., \& Lerner, R.M. (2014). Important non-parental adults and positive youth development across mid- to late- adolescence: The moderating effect of parenting profiles. Journal of Youth \& Adolescence, 43(6), 897-918.

\section{https://doi.org/10.1007/s10964-014-0095-x}

Brewer, B. W., Van Raalte, J. L., \& Linder, D. E. (1993). Athletic identity: Hercules' muscles or Achilles heel? International Journal of Sport Psychology, 24(2), 237254.

Broh, B. (2002). Linking extracurricular programming to academic achievement: Who benefits and why? Sociology of Education, 75(1), 69-95. https://doi.org/10.2307/3090254 
Buford May, R. A. (2001). The sticky situation of sportsmanship: Contexts and contradictions in sportsmanship among high school boys basketball players. Journal of Sport and Social Issues, 25(4), 372-389.

https://doi.org/10.1177/0193723501254003

Camiré, M. (2014). Youth development in North American high school sport: Review and recommendations. Quest, 66(4), 495-511. https://doi.org/10.1080/00336297.2014.952448

Camiré, M. (2015a). Examining high school teacher-coaches' perspective on relationship building with student-athletes. International Sport Coaching Journal, 2(2), 125-136. https://doi.org/10.1123/iscj.2014-0098

Camiré, M. (2015b). Being a teacher-coach in Ontario high schools: Challenges and recommendations. PHEnex Journal, 7(1), 1-15.

Camiré, M. \& Kendellen, K. (2016). Coaching for positive youth development in high school sport. In N. L. Holt (Ed.). Positive Youth Development Through Sport ( $2^{\mathrm{e}}$ éd. p. 126-136). Abingdon: Routledge. https://doi.org/10.4324/9781315709499-11

Camiré, M., Kendellen, K., Rathwell, S., \& Felber Charbonneau, E. (2018). Evaluation of the pilot implementation of the Coaching for Life Skills program. International Sport Coaching Journal, 5(3), 227-236. https://doi.org/10.1123/iscj.2018-0006

Camiré, M., Kendellen, K., Rathwell, S., \& Turgeon, S. (2020). Evaluating the Coaching for Life Skills online training program: A randomised controlled trial. Psychology of Sport and Exercise, 48, 101649. https://doi.org/10.1016/.jpsychsport.2020.101649

Camiré, M., Rocchi, M., \& Kendellen, K. (2016). Profiling the Canadian high school teacher-coach: A national survey. International Sport Coaching Journal, 3(2), 145155. https://doi.org/10.1123/iscj.2015-0110

Camiré, M., Trudel, P., \& Forneris, T. (2009a). Parents' perspectives on the practice of high school sport in a Canadian context. Qualitative Research in Sport and Exercise, 1(3), 239-257. https://doi.org/10.1080/19398440903192324

Camiré, M., Trudel, P., \& Forneris, T. (2009b). High school athletes' perspectives on support, communication, negotiation and life skill development. Qualitative Research in Sport and Exercise, 1(1), 72-88. https://doi.org/10.1080/19398440802673275

Camiré, M., Trudel, P., \& Forneris, T. (2012). Coaching and transferring life skills: Philosophies and strategies used by model high school coaches. The Sport Psychologist, 26(2), 243-260. https://doi.org/10.1123/tsp.26.2.243 
Camiré, M., Rathwell, S., Turgeon, S., \& Kendellen, K. (2019). Coach-athlete relationships, basic psychological needs satisfaction and thwarting, and the teaching of life skills in Canadian high school sport. International Journal of Sports Science \& Coaching, 14(5), 591-606. https://doi.org/10.1177/1747954119869542

Camiré, M., Werthner, P., \& Trudel, P. (2009). Mission statements in sport and their ethical messages: Are they being communicated to practitioners? Athletic Insight, 11(1), 75-85.

Carpentier, J. \& Mageau, G. A. (2016). Predicting sport experience during training: The role of change-oriented feedback in athletes' motivation, self-confidence and needs satisfaction fluctuations. Journal of Sport \& Exercise Psychology, 38(1), 4558. https://doi.org/10.1123/jsep.2015-0210

Carson Sackett, S. \& Gano-Overway, L. A. (2017). Coaching life skills development: Best practices and high school tennis coach exemplar. International Sport Coaching Journal, 4(2), 206-219. https://doi.org/10.1123/iscj.2016-0080

Chinkov, A. E. \& Holt, N. L. (2016). Implicit transfer of life skills through participation in Brazilian Jiu-Jitsu. Journal of Applied Sport Psychology, 28(2), 139-153. https://doi.org/10.1080/10413200.2015.1086447

Clark, H., Camiré, M., Wade, T., \& Cairney, J. (2015). Sport participation and its association with social and psychological factors known to predict substance use and abuse among youth: A scoping review of the literature. International Review of Sport and Exercise Psychology, 8(1), 224-250.

\section{https://doi.org/10.1080/1750984X.2015.1068829}

Coakley, J. (2011). Youth Sports: What counts as "positive development"? Journal of Sport and Social Issues, 35(3), 306-324. https://doi.org/10.1177/0193723511417311

Coakley, J. (2016). Positive youth development through sport: Myths, beliefs, and realities. In N. L. Holt (Ed.), Positive youth development through sport (2e éd. p. 21-31). Abingdon: Routledge.

Coalter, F. (2007). A wider social role for sport: Who's keeping the score? Abingdon: Routledge.

Coalter, F. (2010). The politics of sport-for-development: Limited focus programmes and broad gauge problems? International Review for the Sociology of Sport, 45(3), 295-314. https://doi.org/10.1177\%2F1012690210366791

Collins, K., Gould, D., Lauer, L., \& Chung, Y. (2009). Coaching life skills through football: Philosophical beliefs of outstanding high school football coaches. International Journal of Coaching Science, 3(1), 29-54. 
Crane, J. \& Temple, V. (2015). A systematic review of dropout from organized sport among children and youth. European Physical Education Review, 21(1), 114-131. https://doi.org/10.1177/1356336X14555294

Cunningham, G. B., Dixon, M. A., Singer, J. N., Oshiro, K. F., Ahn, N. Y., \& Weems, A. (2019). A site to resist and persist: Diversity, social justice, and the unique nature of sport. Journal of Global Sport Management, 6(1),1-19.

https://doi.org/10.1080/24704067.2019.1578623

D’Angelo, C., Corvino, C., Cianci, E., \& Gozzoli, C. (2020). Sport for vulnerable youth: The role of multi-professional groups in sustaining intersectoral collaboration. Social Inclusion, 8(3), 129-138. https://doi.org/10.17645/si.v8i3.2745

Danish, S. J., Forneris, T., Hodge, K., \& Heke, I. (2004). Enhancing youth development through sport. World Leisure Journal, 46(3), 38-49.

https://doi.org/10.1080/04419057.2004.9674365

Danish, S. J., Forneris, T., \& Wallace, I. (2005). Sport-based life skills programming in the schools. Journal of Applied School Psychology, 21(2), 41-62.

https://doi.org/10.1300/J370v21n02 04

Danish, S. J., Petitpas, A. J., \& Hale, B. D. (1993). Life development intervention for athletes' life skills through sports. The Counseling Psychologist, 21(3), 352-385. https://doi.org/10.1177/0011000093213002

DiFiori, J. P., Benjamin, H. J., Brenner, J., Gregory, A., Jayanthi, N, Landry, G. L., \& Luke, A. (2014). Overuse injuries and burnout in youth sports: A position statement from the American Medical Society for Sports Medicine. Clinical Journal of Sport Medicine, 24(1), 3-20. https://doi.org/10.1097/JSM.0000000000000060

Durlak, J. A., Weissberg, R. P., \& Pachan, M. (2010). A meta-analysis of after-school programs that seek to promote personal and social skills in children and adolescents. American Journal of Community Psychology, 45(3-4), 294-309. https://doi.org/10.1007/s10464-010-9300-6

Dworkin, J., \& Larson, R. W. (2006). Adolescents' negative experiences in organized youth activities. Journal of Youth Development, 1(3), 1-18.

https://doi.org/10.5195/jyd.2007.373

Eccles, J. S. \& Barber, B. L. (1999). Student council, volunteering, basketball, or marching band: What kind of extracurricular involvement matters? Journal of Adolescent Research, 14(1), 10-43. https://doi.org/10.1177/0743558499141003 
Eccles, J. S., Barber, B. L., Stone, M., \& Hunt, J. (2003). Extracurricular activities and adolescent development. Journal of Social Issues, 59(4), 865-889. https://doi.org/10.1046/j.0022-4537.2003.00095.x

Ferris, K. A., Ettekal, A. V., Agans, J. P., \& Burkhard, B. M. (2016). Character development through youth sport: High school coaches' perspectives about a character-based education program. Journal of Youth Development, 10(3), 127-140. https://doi.org/10.5195/JYD.2015.13

Fox, C. K., Barr-Anderson, D., Neumark-Sztainer, D., \& Wall, M. (2010). Physical activity and sports team participation: Associations with academic outcomes in middle school and high school students. Journal of School Health, 80(1), 31-37. https://doi.org/10.1111/j.1746-1561.2009.00454.x

Fraser-Thomas, J. \& Côté, J. (2009). Understanding adolescents' positive and negative developmental experiences in sport. The Sport Psychologist, 23(1), 3-23. https://doi.org/10.1123/tsp.23.1.3

Fraser-Thomas, J., Côté, J., \& Deakin, J. (2005). Youth sport programs: An avenue to foster positive youth development. Physical Education and Sport Pedagogy, 10(1), 19-40. https://doi.org/10.1080/1740898042000334890

Fuller, R. \& Agyemang, K. (2018). An examination of activism and NCAA Division III Black male athletes. International Journal of Sport Management, 19, 186-206.

Geldhof, G. J., Bowers, E. P., Johnson, S. K., Hershberg, R., Hilliard, L., Lerner, J. V., \& Lerner, R. M. (2013). Relational developmental systems theories of positive youth development: Methodological issues and implications. In P. C. M. Molenaar, R. M. Lerner, \& K. M. Newell (Eds.), Handbook of developmental systems theory and methodology (p. 66-94). New York: The Guilford Press.

Gould, D. \& Carson, S. (2008). Life skills development through sport: Current status and future directions. International Review of Sport and Exercise Psychology, 1(1), 5878. https://doi.org/10.1080/17509840701834573

Gould, D., Collins, K., Lauer, L., \& Chung, Y. (2007). Coaching life skills through football: A study of award winning high school coaches. Journal of Applied Sport Psychology, 19(1), 16-37. https://doi.org/10.1080/10413200601113786

Gould, D., Chung, Y., Smith, P., \& White, J. (2006). Future directions in coaching life skills: Understanding high school coaches' views and needs. Athletic Insight, 8(3), 28-38.

Gould, D. \& Westfall, S. (2013). Promoting life skills in children and youth: Applications to sport contexts. In A. R. Gomes, R. Resende, \& A. Albuquerque (Eds.), Positive 
human functioning from a multi-dimensional perspective. Vol. 2: Promoting healthy lifestyles (p. 53-77). New York: Nova Publishing.

Green, K., Nelson, T., \& Hartmann, D. (2014). Binge drinking and sports participation in college: Patterns among former athletes and athletes. International Review for the Sociology of Sport, 49(3-4), 417-434. https://doi.org/10.1177/1012690213509257 Hager, P., \& Hodkinson, P. (2009). Moving beyond the metaphor of transfer of learning. British Educational Research Journal, 35(4), 619-638.

\section{https://doi.org/10.1080/01411920802642371}

Harrison, P. A. \& Narayan, G. (2003). Differences in behavior, psychological factors, and environmental factors associated with participation in school sports and other activities in adolescence. Journal of School Health, 73(3), 113-120.

https://doi.org/10.1111/j.1746-1561.2003.tb03585.x

Harwood, C. G., Barker, J. B., \& Anderson, R. (2015). Psychosocial development in youth soccer players: Assessing the effectiveness of the $5 \mathrm{Cs}$ intervention program. The Sport Psychologist, 29(4), 319-334. https://doi.org/10.1123/tsp.2014-0161

Hennig, L. C., Schaefer, L., \& Gleddie, D. (2020). In(di)visable: Inquiring into being 'othered' as a means to teach social justice in PHETE. Physical Education and Sport Pedagogy. https://doi.org/10.1080/17408989.2020.1789573

Heroux D. \& Strashin, J. (2020). Sidelined: How diversity in Canada's sports leadership falls short [en ligne]. Repéré à https://www.cbc.ca/sports/diversity-canadiansports-leadership-falls-short-1.5648402

Ho, F. K. W., Louie, L. H. T., Wong, W. H. S., Chan, K. L., Tiwari, A., Chow, C. B., Ho, W., Wong, W., Chan, M., Chen, E. Y. H., Cheung, Y. F., \& Ip, P. (2017). A sportsbased youth development program, teen mental health, and physical fitness: An RCT. Pediatrics, 140(4), 1-10. https://doi.org/10.1542/peds.2017-1543

Hodge, K. \& Danish, S. J. (1999). Promoting life skills for adolescent males through sport. In A. Horne, \& M. Kiselica (Eds.), Handbook of counseling boys and adolescent males (p. 55-71). Thousand Oaks: Sage.

Hodge, K., Danish, S., \& Martin, J. (2013). Developing a conceptual framework for life skills interventions. The Counseling Psychologist, 41(8), 1125-1152.

https://doi.org/10.1177/0011000012462073

Holt, N. L. (Ed.) (2016). Positive youth development through sport. Abingdon: Routledge.

Holt, N. L. \& Neely, K. C. (2011). Positive youth development through sport: A review. Revista de Iberoamericana de Psicología del Ejercicio y el Deporte (Version anglaise), 6(2), 299-316. 
Holt, N. L., Tink, L. N., Mandigo, J. L., \& Fox, K. R. (2008). Do youth learn life skills through their involvement in high school sport? A case study. Canadian Journal of Education, 31(2), 281-304. https://www.jstor.org/stable/20466702

Jewett, R., Sabiston, C. M., Brunet, J., O’Loughlin, E. K., Scarapicchia, T., \& O’Loughlin, J. (2014). School sport participation during adolescence and mental health in early adulthood. Journal of Adolescent Health, 55(5), 640-644.

https://doi.org/10.1016/i.jadohealth.2014.04.018

Jones, G. J., Edwards, M. B., Bocarro, J. N., Svensson, P. G., Misener, K. (2020). A community capacity building approach to sport-based youth development. Sport Management Review, 23(4), 563-575. https://doi.org/10.1016/j.smr.2019.09.001

Jones, M. I. \& Lavallee, D. (2009). Exploring perceived life skills development and participation in sport. Qualitative Research in Sport and Exercise, 1(1), 36-50. https://doi.org/10.1080/19398440802567931

Jowett, S. (2007). Interdependence analysis and the 3+1Cs in the coach-athlete relationship. In S. Jowett \& D. Lavallee (Eds.), Social psychology in sport (p. 1528). Champaign, IL: Human Kinetics.

Kendellen, K. \& Camiré, M. (2015a). Examining former athletes' developmental experiences in high school sport. SAGE Open, 1-10. https://doi.org/10.1177/2158244015614379

Kochanek, J. \& Erickson, K. (2020). Interrogating positive youth development through sport using critical race theory. Quest, 72(2), 224-240. https://doi.org/10.1080/00336297.2019.1641728

Kulick, A., Wernick, L., Espinoza, M., Newman, T., \& Dessel, A. (2019). Three strikes and you're out: Culture, facilities, and participation among LGBTQ youth in sports. Sport, Education and Society, 24(9), 939-953.

https://doi.org/10.1080/13573322.2018.1532406

Kwan, M., Bobko, S., Faulkner, G., Donnelly, P., \& Cairney, J. (2014). Sport participation and alcohol and illicit drug use in adolescents and young adults: A systematic review of longitudinal studies. Addictive Behaviors, 39(3), 497-506.

https://doi.org/10.1016/j.addbeh.2013.11.006

Lacroix, C., Camiré, M., \& Trudel, P. (2008). High school coaches' characteristics and their perspectives on the purpose of school sport participation. International Journal of Coaching Science, 2(2), 23-42.

Lafont, L. (2002). Technique, modèles et didactique de l'éducation physique et sportive. STAPS, 59(3), 57-70. https://doi.org/10.3917/sta.059.0057 
Larson, R. (2000). Toward a psychology of positive youth development. American Psychologist, 55(1), 170-183. https://doi.org/10.1037//0003-066x.55.1.170

Leavey, G. \& Breslin, G. (2019). Mental health and well-being interventions in sport: Case studies and analysis. Abingdon: Routledge.

Leberman, S., McDonald, L., \& Doyle, S. (2006). The transfer of learning: Participants' perspectives of adult education and training. Farnham, Surrey, UK : Gower Publishing.

Lee, O. \& Martinek, T. (2013). Understanding the transfer of values-based youth sport program goals from a bioecological perspective. Quest, 65(3), 300-312. https://doi.org/10.1080/00336297.2013.791871

Lerner, R.M., Almerigi, J., Theokas, C., \& Lerner, J. (2005). Positive youth development: A view of the issues. The Journal of Early Adolescence, 25(1), 10-16. https://doi.org/10.1177/0272431604273211

Lerner, R. M. (2002). Adolescence: Development, diversity, context, and application. Saddle River, NJ : Prentice Hall/Pearson Education.

Mac Intosh, A., Martin, E. M., \& Kluch, Y. (2020). To act or not to act? Student-athlete perceptions of social justice activism. Psychology of Sport \& Exercise. 51, 101766. https://doi.org/10.1016/j.psychsport.2020.101766

Macquet, A. C. \& Fleurance, P. (2006). Des modèles théoriques pour étudier l'activité de l'expert en sport. Science et Motricité, 58, 9-41. https://doi.org/10.3917/sm.058.09 Mahoney, J. (2000). School extracurricular activity participation as a moderator in the development of antisocial patterns. Child Development, 71(2), 502-516. https://doi.org/10.1111/1467-8624.00160

Marsh, H. W. \& Kleitman, S. (2003). School athletic participation: Mostly gain with little pain. Journal of Sport \& Exercise Psychology, 25(2), 205-228.

https://doi.org/10.1123/jsep.25.2.205

National Collegiate Athletic Association. (2018). Estimated probability of competing in college athletics. Repéré à http://www.ncaa.org/about/resources/ research/estimated-probability-competing-college-athletics

Neely, S. R. \& Vaquera, E. (2017). Making it count: Breadth and intensity of extracurricular engagement and high school dropout. Sociological Perspective, 60(6), 1039-1062. https://doi.org/10.1177/0731121417700114

Newman, T., Lower-Hoppe, L. M., Burch, M., \& Paluta, L. M. (2020). Advancing positive youth development-focused coach education: Contextual factors of youth sport 
and youth sport leader perceptions. Managing Sport and Leisure, 1-15 https://doi.org/10.1080/23750472.2020.1766760

Newman, T., Okamoto, K., Kimiecik, C., Sohns, E., Burns, M., \& Magier, E. (2019). The role of social workers in sport: Shared values, interprofessional collaborations, and unique contributions. Journal of Sport Psychology in Action, 10(3), 160-173. https://doi.org/10.1080/21520704.2019.1642270

Newman, T., Ortega, R., Lower, L., \& Paluta, L. (2016). Informing priorities for coaching education: Perspectives from youth sport leaders. International Journal of Sports Science \& Coaching, 11(3), 436-445. https://doi.org/10.1177/1747954116645207

Petitpas, A. J., Cornelius, A. E., Van Raalte, J. L., \& Jones, T. (2005). A framework for planning youth sport programs that foster psychosocial development. The Sport Psychologist, 19(1), 63-80. https://doi.org/10.1123/tsp.19.1.63

Pierce, S., Gould, D., \& Camiré, M. (2017). Definition and model of life skills transfer. International Review of Sport and Exercise Psychology, 10(1), 186-211. https://doi.org/10.1080/1750984X.2016.1199727

Pierce, S., Gould, D., Cowburn, I., \& Driska, A. (2016). Understanding the process of psychological development in youth athletes attending an intensive wrestling camp. Qualitative Research in Sport, Exercise and Health, 8(4), 332-351. https://doi.org/10.1080/2159676X.2016.1176067

Pierce, S., Kendellen, K., Camiré, M., \& Gould, D. (2018). Strategies for coaching life skills transfer. Journal of Sport Psychology in Action, 9(1), 11-20. https://doi.org/10.1080/21520704.2016.1263982

Ronkainen, N. J., Aggerholm, K., Ryba, T. V., \& Allen-Collinson, J. (2020): Learning in sport: From life skills to existential learning. Sport, Education and Society, 26(2), 214-227. https://doi.org/10.1080/13573322.2020.1712655

Ryan, R.M. \& Deci, E.L. (2000). The darker and brighter sides of human existence: Basic psychological needs as a unifying concept. Psychological Inquiry, 11(4), 319-338. https://doi.org/10.1207/S15327965PLI1104 03

Savery, J.R. (2015). Overview of problem-based learning: Definitions and distinctions. In A. Walker, H. Leary, C. Hmelo-Silver, \& P.A. Ertmer (Eds.), Essential readings in problem-based learning (p. 5-15). West Lafayette, IN: Purdue University.

Scanlan, T., Babkes, M., \& Scanlan, L. (2005). Participation in sport: A developmental glimpse at emotion. In J. Mahoney, J. Eccles, \& R. Larson (Eds.), After school activities: Contexts of development. Mahwah, NJ : Lawrence Erlbaum.

School Sport Canada (2018). About SSC. Repéré à http://www.schoolsport.ca/ 
Simard, S. (2014). Le développement positif des jeunes en contexte sportif parascolaire : évaluation du programme d'intervention psychosociale 'Bien dans mes Baskets'. Thèse de doctorat non publiée, Université de Montréal, Montréal. Repéré à http://hdl.handle.net/1866/10533

Smith, R. E., Smoll, F. L., \& Barnett, N. P. (1995). Reduction of children's sport performance anxiety through social support and stress-reduction training for coaches. Journal of Applied Developmental Psychology, 16(1), 125-142. https://doi.org/10.1016/ 0193-39739590020-9

Smith, R. E., Smoll, F. L., \& Cumming, S. P. (2007). Effects of a motivational climate intervention for coaches on young athletes' sport performance anxiety. Journal of Sport \& Exercise Psychology, 29(1), 39-59. https://doi.org/10.1123/jsep.29.1.39

Smoll, F. L. \& Smith, R. E. (1996). Children and youth in sport: A biopsychosocial perspective. Dubuque, $1 \mathrm{O}:$ Kendall Hunt.

Snyder, C. R. \& Lopez, S. J. (2002). Handbook of positive psychology. Oxford: Oxford University Press.

Spaaij, R., Knoppers, A., \& Jeanes, R. (2020). "We want more diversity but...": Resisting diversity in recreational sports clubs. Sport Management Review, 23, 363-373. https://doi.org/10.1016/j.smr.2019.05.007

Steiner, H., McQuivey, R. W., Pavelski, R., Pitts, T., \& Kraemer, H. (2000). Adolescents and sports: Risk or benefit? Clinical Pediatrics, 39(3), 161-166. https://doi.org/10.1177/000992280003900304

Strachan, L., MacDonald, D. J., \& Côté, J. (2016). Project SCORE! Coaches' perceptions of an online tool to promote positive youth development in sport. International Journal of Sports Science \& Coaching, 11(1), 108-115. https://doi.org/10.1177/ 1747954115624827

Trottier, C., \& Robitaille, S. (2014). Fostering life skills development in high school and community sport: A comparative analysis of the coach's role. The Sport Psychologist, 28(1), 10-21. https://doi.org/10.1123/tsp.2012-0094

Turgeon, S., Camiré, M., \& Rathwell, S. (2020). Follow-up evaluation of the Coaching for Life Skills online training program. International Journal of Sports Science \& Coaching, 16 (1), 173-180. https://doi.org/10.1177/1747954120964075

Van Boekel, M., Bulut, O., Stanke, L., Palma Zamora, J. R., Jang, Y., Kang, Y., \& Nickodem, K. (2016). Effects of participation in school sports on academic and social functioning. Journal of Applied Development Psychology, 46, 31-40. https://doi.org/10.1016/..appdev.2016.05.002 
Vella, S. A., Oades, L. G., \& Crowe, T. P. (2013). A pilot test of transformational leadership training for sports coaches: Impact on the developmental experiences of adolescent athletes. International Journal of Sports Science \& Coaching, 8(3), 513-530. https://doi. org/10.1260/1747-9541.8.3.513

Walton-Fisette, J. L. \& Sutherland, S. (2018). Moving forward with social justice education in physical education teacher education. Physical Education and Sport Pedagogy, 23(5), 461-468. https://doi.org/10.1080/17408989.2018.1476476

Weiss, M. R. \& Wiese-Bjornstal, D. M. (2009). Promoting positive youth development through physical activity. President's Council on Physical Fitness and Sports Research Digest, 10(3), 1-8. 